\title{
Bosonic versus fermionic pairs of topological spin defects in monolayered high- $T_{c}$ superconductors
}

\author{
M. A. Garcia-Bach* \\ Departament de Física Fonamental, Facultat de Física, Universitat de Barcelona, Diagonal 647, E-08028 Barcelona, Catalunya, Spain
}

(Received 28 October 2004; revised manuscript received 20 April 2005; published 18 July 2005)

\begin{abstract}
The energy associated with bosonic and fermionic pairs of topological spin defects in doped antiferromagnetic quantum spin-1/2 square lattice is estimated within a resonating valence bond scenario, as described by a $t$ - $t^{\prime}$ - $J$-like model Hamiltonian, plus a $t_{\perp}$, responsible of a three-dimensional screening of the electrostatic repulsion within the bosonic pairs. For parameters appropriate for monolayered high- $T_{c}$ superconductors, both fermionic and bosonic pairs show $x^{2}-y^{2}$ symmetry. We find a critical value of doping such that the energy of the bosonic pairs goes below twice the energy of two fermionic pairs at their Fermi level. This finding could be related to the onset of high- $T_{c}$ superconductivity.
\end{abstract}

DOI: 10.1103/PhysRevB.72.024530

PACS number(s): 74.20.Mn, 71.27.+a, 75.10.Jm

\section{INTRODUCTION}

Since the discovery of high- $T_{c}$ superconductivity (HTSC) in $\mathrm{La}_{2} \mathrm{CuO}_{4}$, ${ }^{1}$ a vast amount of work has been done on slightly doped quasi-two-dimensional (2D) antiferromagnets. ${ }^{2-6}$ It has been observed that these materials display very unusual properties, with a rich variety of temperaturedoping phases diagram. Specifically, away from the overdoped side, the cuprates do not appear to be a Landau Fermi liquid. For instance, they should be considered as doped Mott insulators. However, the theoretical status of the field has been largely phenomenological and controversial. ${ }^{5,6}$ As far as we know, there is no consensus on the origin of the superconductivity nor on the pseudogap phase. Therefore, finding a microscopic mechanism for HTSC still is an open problem.

Local-density approximation (LDA) and generalized gradient approximation $(\mathrm{GGA})^{7}$ to density functional theory have been used so far to rationalize the electronic structure of HTSCs. Although LDA is a useful technique for some materials, it has been shown that both LDA or GGA are not appropriate for antiferromagnetic materials because they tend to yield a metallic ground state with incorrect delocalized spin density and band ordering. ${ }^{8,9}$ This is attributed to an extreme nonanalytic and nonlocal behavior of density functional theory as the particle number is changed, ${ }^{10,11}$ implying the need for a self-energy correction, or at least an orbital dependent potential to obtain a realistic description of band gaps. To overcome such a problem, different semiempirical corrections to LDA have been proposed so far as, i.e., LDA + SIC (Refs. 12-14) and LDA $+U .^{15-17}$

An alternative approach to the electronic structure of the HTSCs is based on the use of model Hamiltonians that aim to incorporate the essential physics into a few parameters. It is generally accepted that electron correlation is important for HTSC. Furthermore, it is well known that the (covalentstructure) valence-bond (VB) model or, equivalently, the Heisenberg Hamiltonian includes most of the electron correlation. Thence, early in 1987, Anderson ${ }^{18}$ proposed that the important features of the undoped HTSC parent compounds can be described by a Heisenberg Hamiltonian on a twodimensional square lattice with one electron per site. Meanwhile, Emery ${ }^{19,20}$ proposed a three-band Hubbard model.
Unfortunately, the number of parameters of a three-band Hubbard model turns to be too large. Therefore, Zhang and Rice $^{21}$ proposed a simplification of the three-band Hubbard model into the well known $t$ - $J$, which implicitly includes the $\mathrm{O}(p)-\mathrm{Cu}(d)$ hybridization and recovers the initial effective one-band description of Anderson. Since an appropriate parametrization is essential for the predictive capability of model Hamiltonians, much progress has been achieved on the high-level $a b$ initio computation of reliable appropriate parameters using only the crystal structure as external input. $^{22-24}$

Stimulated by Anderson's suggestion, ${ }^{18}$ a renewed interest of low-dimensional quantum spin-1/2 antiferromagnetic systems emerged. According to the Lieb and Mattis theorem ${ }^{25}$ the ground state for the undoped half-filled bipartite system must be a singlet. Therefore, the appropriate ground-state wave function could have a resonating-valence-bond (RVB) character. It was soon pointed out that short-range RVB wave functions exhibit topological long-range order. ${ }^{26-29}$ Furthermore, recently ${ }^{30}$ topological order for superconductors has also been claimed, away from truly microscopic models, making use of bosonic theories of the quantum GinzburgLandau form. In Ref. 28 Klein and collaborators investigated the short-range RVB wave functions within a dimer coverings approximation for the square lattice and found that the dimer coverings show a type of long-range spin-pairing order (LRSPO). Using arguments based on the LRSPO they predicted a per-site energy $\varepsilon \propto \delta^{2}$, where $\delta$ is the deviation of the local LRSPO with respect to the LRSPO of the ground state. Furthermore, topological spin defects (TSDs), namely a site that is not spin paired to a singlet, or a hole in holedoped superconductors, or a doubly occupied site in electron-doped materials, were assimilated to Bloch walls separating phases with a difference in LRSPO of \pm 1 . It was argued that, at a longitudinal distance $\sim \rho$ past the TSD on the less stabilized side, the defect should also presumably have only spread out a transverse distance $\sim \rho$, so that $\delta$ $\sim 1 / \rho$, and $\varepsilon \propto 1 / \rho^{2}$. Therefore, the energy contribution from all the sites of the given longitudinal distance past the TSD is $\rho \Delta \varepsilon \sim 1 / \rho$. Summation over all the sites up to a given distance thence gives an energy cost of $\sim \ln \rho$. When the TSD are charged, it was also suggested that this long flat attraction 
$\sim \ln \rho$ along with the screened repulsion $\exp \{-\alpha \rho\} / \rho$ could lead to a weakly bound pair. Recently, ${ }^{22}$ a linear relationship between $T_{c}$ and the $J / t$ ratio, as obtained from high-level $a b$ initio calculations, was found. It was argued that such a linear relation arises from the LRSPO mechanism previously suggested. ${ }^{28}$ Furthermore, the so-called $t-J$ Hamiltonian for the cuprates seems to be pointing to the right direction.

The existence of a LRSPO for more general RVB wave functions has been proven for ladderlike quantum spin-1/2 antiferromagnetic systems. ${ }^{31,32}$ Most of the considerations associated to the existence of this LRSPO for the ladderlike quantum spin-1/2 antiferromagnetic systems are readily applicable to the square lattice. In particular, bound pairs of TSD are predicted to occur. However, as far as we know, the energy of such a pair of TSD as a function of the distance has not been obtained yet. Even more, arguments based on the LRSPO alone cannot decide if vacancies (doubly occupied sites), let's say charge-wearing TSDs, organize themselves as bound pairs of two charge-wearing TSDs, as bosoniccharacter pairs, or each charge-wearing TSD would bind to a non-spin-paired spin, let us say a spin-wearing TSD, leading to a fermionic-character pair.

Here focus is on the energy associated with these bosonic and fermionic pairs as described by symmetry-adapted extended wave functions. We find that the fermionic pairs are favored for low doping levels, but the Fermi level increases with doping while the energy of the bosonic pairs lowers. At a critical doping the energy of the bosonic pair goes below the energy of two fermionic pairs at the Fermi level, suggesting the pairing of charge-wearing TSDs, and hence providing a microscopic mechanism for HTSC.

The description of these bosonic and fermionic pairs is based on a $t-t^{\prime}-J$-like model Hamiltonian $H=H_{I}+H_{J}+H_{t}$ $+H_{t^{\prime}}$, where $H_{I}$ is the energy associated with the ionization potential for hole-doped materials, or the energy associated with the electron affinity for the electron-doped systems. The $H_{J}$ is the well known nearest-neighbor Heisenberg Hamiltonian,

$$
H_{J}=J \sum_{\left\langle\mathbf{R}, \mathbf{R}^{\prime}\right\rangle} \mathbf{S}_{\mathbf{R}} \cdot \mathbf{S}_{\mathbf{R}^{\prime}},
$$

where $\mathbf{S}_{\mathbf{R}}$ is the spin operator for the spin on the site $\mathbf{R}$, and $\left\langle\mathbf{R}, \mathbf{R}^{\prime}\right\rangle$ means that $\mathbf{R}$ and $\mathbf{R}^{\prime}$ are nearest neighbors. The nearest- and next-nearest-neighbor hopping contributions to the Hamiltonian are, respectively,

$$
H_{t}=-t \sum_{\left\langle\mathbf{R}, \mathbf{R}^{\prime}\right\rangle} \sum_{\sigma}\left(c_{\mathbf{R} \sigma}^{\dagger} c_{\mathbf{R}^{\prime} \sigma}+c_{\mathbf{R}^{\prime} \sigma}^{\dagger} c_{\mathbf{R} \sigma}\right) \times\left(1-\hat{n}_{\mathbf{R} \bar{\sigma}}\right)\left(1-\hat{n}_{\mathbf{R}^{\prime} \sigma}\right),
$$

$$
\begin{aligned}
H_{t^{\prime}}= & \sum_{\left\langle\left\langle\mathbf{R}, \mathbf{R}^{\prime}\right\rangle\right\rangle} t_{\left\langle\left\langle\mathbf{R}, \mathbf{R}^{\prime}\right\rangle\right\rangle}^{\prime} \sum_{\sigma}\left(c_{\mathbf{R} \sigma}^{\dagger} c_{\mathbf{R}^{\prime} \sigma}+c_{\mathbf{R}^{\prime} \sigma^{\prime}}^{\dagger} c_{\mathbf{R} \sigma}\right) \\
& \times\left(1-\hat{n}_{\mathbf{R} \bar{\sigma}}\right)\left(1-\hat{n}_{\mathbf{R}^{\prime} \bar{\sigma}}\right),
\end{aligned}
$$

where $c_{\mathbf{R} \sigma}^{\dagger}\left(c_{\mathbf{R} \sigma}\right)$ creates (destroys) an electron on site $\mathbf{R}$ with spin $\sigma=\alpha, \beta$. The double occupancy is avoided by the factors $1-\hat{n}_{\mathbf{R} \bar{\sigma}}$, where $\hat{n}_{\mathbf{R} \bar{\sigma}}$ is the number operator on site $\mathbf{R}$ with spin $\bar{\sigma}=\beta, \alpha$. The summation on $\left\langle\left\langle\mathbf{R}, \mathbf{R}^{\prime}\right\rangle\right\rangle$ means that $\mathbf{R}$ and
$\mathbf{R}^{\prime}$ are next-nearest neighbors. The hopping integral ${ }^{*} t_{\left\langle\left\langle\mathbf{R}, \mathbf{R}^{\prime}\right\rangle\right\rangle}$ depends on the number of holes within the plaquette. Such a model is known to reproduce the low-energy spectrum of the three-band Hubbard model. ${ }^{33}$ Here, we use the parameters obtained by high-level ab initio calculations using only the crystal structure as external input. ${ }^{22,23}$ We approximate the screened electrostatic repulsion within the bosonic pair by the Yukawa potential, ${ }^{34,35}$ the screening agent being the gas of the fermionic pairs. The three-dimensional (3D) character of the screening is taken into account by an interlayer hopping integral $t_{\perp}$. The Heisenberg part of the energy associated with a pair of static TSDs is estimated by the dimer-covering-counting approximation ${ }^{36-38}$ on $w \times L$ antiferromagnetic quantum spin-1/2 square lattice, with $w$ $=4,6, \ldots, 20, L \rightarrow \infty$, and cyclic boundary conditions in both directions. Counting of the dimer-covering configurations has been achieved by a transfer-matrix technique. ${ }^{31,32}$

This paper is organized as follows: In Sec. II we review the main concepts about LRSPO, ${ }^{31,32}$ describing the scenario where the TSDs are located. In Sec. III the energy per site of the half-filled ground state, and the gain of the Heisenberg energy associated with a static pair of TSDs is estimated. In Sec. IV the symmetry-adapted extended wave-functions appropriate for bosonic and fermionic pairs moving in a $\mathrm{CuO}_{2}$ layer will be defined, and the energy bands will be obtained. From the two-fluids equilibrium condition, the critical doping $p_{c}$ for the onset of pairing to bosonic pairs among chargewearing TSDs is obtained. Finally a summary and the conclusions are given in Sec. V.

\section{LONG-RANGE SPIN-PAIRING ORDER AND TOPOLOGICAL SPIN DEFECTS}

From the Lieb and Mattis theorem ${ }^{25}$ it is well known that for bipartite spin systems a maximally-spin-paired ground state is expected. In particular, at half-filling, for ladderlike systems, with equal number of sites in the $\mathcal{A}$ and $\mathcal{B}$ sublattices, the ground state is a singlet. Singlet states can be achieved by configuration interaction (CI) among covalent VB configurations or RVB. For instance, a linearly independent set of VB configurations can be achieved by pairing to a singlet each spin in the sublattice $\mathcal{A}$ to a spin in the sublattice $\mathcal{B}$, not necessarily one of its nearest neighbors (see Fig. 1).

It is known ${ }^{31,32}$ that any (covalent) VB configuration exhibits a LRSPO related to the local (at boundary) array of SPs penetrating any boundary $f_{n}$ (see, for instance, Fig. 1). The parameter associated with the LRSPO, $D$, can take $w$ +1 different relevant values. The shape of the boundary selected to define the different $w+1$ values of $D$ is quite arbitrary, though when $w=$ even and the boundary is chosen to run parallel to rungs, the $w+1$ different values of $D$ are

$$
D=0, \pm 1, \ldots, \pm \frac{w}{2} .
$$

This LRSPO allows to separate the set of VB configurations in different subsets. Since two singlets from different subsets must be different repeatedly at every position along the ladder, they are asymptotically orthogonal and noninteracting 


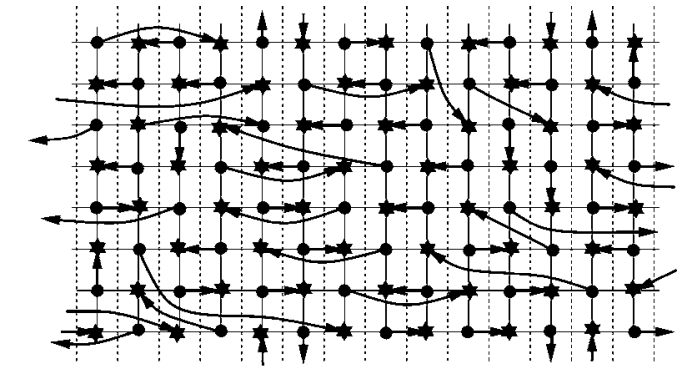

$\mathrm{D}=0 \begin{array}{lllllllllllllll}0 & 0 & 0 & 0 & 0 & 0 & 0 & 0 & 0 & 0 & 0 & 0 & 0 & 0 & 0\end{array}$

FIG. 1. A fragment of a VB configuration for a $w=8$ square lattice. Each arrow represents a spin pairing (SP) to a singlet between a spin on a site in the sublattice $\mathcal{A}$ (circles) with a spin on a site in the sublattice $\mathcal{B}$ (stars). Below each boundary, i.e., the dashed lines running parallel to the rungs, it appears the net count of arrows, $D$, penetrating this boundary.

via any interaction mediated by a few-particle operator. Then the matrix of the Hamiltonian asymptotically block diagonalizes, so configurations belonging to different subsets do not mix in the CI sense. Thus $D$ may be taken as a long-range order parameter labelling the eigenstates of the $D$ block. Under low-frustration conditions, the expected ordering of the lowest-lying energy $E_{D}$ from the different blocks is

$$
E_{0}<E_{1}<\cdots<E_{w / 2}
$$

with $E_{D}=E_{-D}$.

Now, half-filled excited states or slightly doped states are analyzed via TSDs. There are different types of excitations conceivable from a maximally-spin-paired ground state. Let us say, preserving half-filling (one electron per site), there are primarily spin excitations. In this case, two spin-wearing TSDs, one in the sublattice $\mathcal{A}$ and the other in the sublattice $\mathcal{B}$, are obtained by breaking one SP to form a triplet state. Away from half-filling, there are low-energy spin and charge excitations. For instance, removing (adding) one electron produces two sites that cannot be SP, a charge-wearing TSD and a spin-wearing TSD, one in the sublattice $\mathcal{A}$ and the other in the sublattice $\mathcal{B}$, the ladder becoming a doublet. In this case hopping terms must be retained in the Hamiltonian and the so-called $t$ - $J$ model or different extensions that incorporate either next-nearest-neighbor hopping $t^{\prime}$ or electrostatic repulsion have been employed so far. Thence, the doublet is a weighted superposition of VB configurations with a spin-wearing TSD and a charge-wearing TSD lying in different sublattices. Still, going up in the hierarchy of Hamiltonians, the Hubbard or even a more general Hamiltonian must be considered. In this case, still another type of excitations (though presumably of higher energy if a Heisenberg-type Hamiltonian is assumed to govern the lowest-lying region of the spectrum) can be produced relaxing the single-occupancy constrain. This leads to the ionic states, i.e., states with at least a pair of sites, one doubly occupied and the other empty, namely one negatively charge-wearing TSD and one positively charge-wearing TSD.

Of special relevance here is how the LRSPO is disrupted by a TSD (see Fig. 2). For instance, a TSD in a site $[n, i], n$ indicating the rung and $i$ the leg, can be seen as a domain

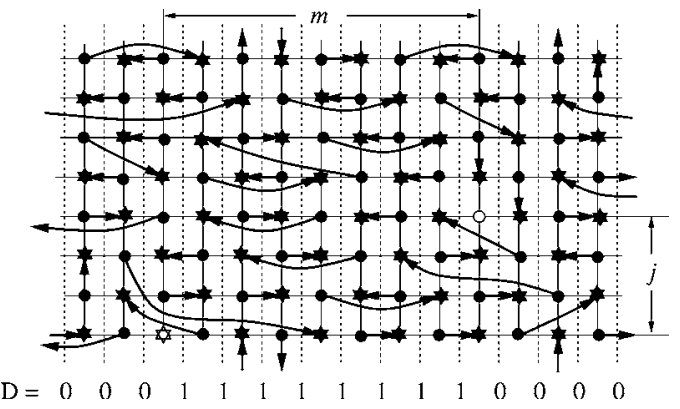

FIG. 2. A fragment of a VB configuration for a $w=8$ square lattice containing a pair of TSDs, one in the sublattice $\mathcal{A}$ (white circle), and the other in the sublattice $\mathcal{B}$ (white star). Notice that this VB configuration shows LRSPO $D=0$ everywhere but in the intervening region defined by the pair of TSDs, with $D=1$.

wall on the rung $n$ which separates the lattice in two sectors with associated left, $D_{l}$, and right, $D_{r}$, order parameters. When we choose the sublattice $\mathcal{A}$ as formed by the set of sites $[m, j]$ with $m+j=$ even,

$$
D_{r}=D_{l}-(-1)^{n+i}
$$

Thence, to fulfill boundary conditions TSDs must appear by pairs, one TSD in the sublattice $\mathcal{A}$ and the other in the sublattice $\mathcal{B}$, to ensure $\Delta D=0$ from the left to the right of the pair. Such a pair define an intervening region with $\Delta D= \pm 1$ with respect to the LRSPO $D$ of the host (see Fig. 2). Then, away from half-filling, it may be conceivable an intervening region limited by two charge-wearing TSDs, or a chargewearing TSD and a spin-wearing TSD (provided that the doping is not so strong as to preclude a maximally spinpaired ground state). In particular, when placing a pair of TSDs above the ground state $(D=0)$, the order parameter of the intervening region will be $\left|D_{p}\right|=1$, which from Eq. (5) is expected to have higher associated energy per site. This indicates that the pair of TSDs should try to remain as close as possible. Thus, bound pairs of TSDs are predicted to occur. To show that this is the case is one of the concerns of the present paper.

\section{HEISENBERG ENERGY OF A STATIC PAIR OF TSDS}

Within the dimer-covering-counting approximation the resonance energy, $E_{r}(w, D)$ in units of $J$, i.e., the energy correction below the energy of a single dimer-covering structure, depends on the configuration interaction among the different dimer-covering configurations with LRSPO $D$. When an equally weighted wave function is considered, it has been $\operatorname{argued}^{36-38}$ that one might consider this energy lowering to depend solely on the dimension of the space spanned by the appropriate dimer-covering configurations. Let $\mathcal{N}_{D}(w)$ be the number of linearly independent dimer-covering configurations with the LRSPO $D$. Since $\mathcal{N}_{D}(w)$ is multiplicative in terms of a break up into subsystems while the energy is additive, such a functional dependence should be of the form

$$
E_{r}(w, D) \approx-C \ln \mathcal{N}_{D}(w) .
$$


The values $\mathcal{N}_{D}(w)$ can be easily obtained by a transfermatrix technique. ${ }^{31}$ Let us start computing $\mathcal{N}_{D}(w)$ for a maximally spin-paired half-filled system. Let us analyze from a local point of view the dimer-covering singlets. We can identify the dimer-covering local states according to which legs have a pairing across the $f_{n}$ boundary. In the present case it can be seen that, for each boundary, there are $2^{w}$ different local states, $\left.\mid e_{n I}\right)$ ( $I$ ranging), which can be classified according to the value of $\left.D, \mid e_{n I}^{D}\right)$.

Proceeding from the left to the right, from the boundary $f_{n-1}$ to $f_{n}$, a dimer-covering-counting matrix, $\mathcal{K}_{n}$, is defined as $\left(e_{n-1 I}\left|\mathcal{K}_{n}\right| e_{n J}\right) \equiv$ the number of different ways $\left.\mid e_{n J}\right)$ can succeed $\left.\mid e_{n-1 I}\right)$. Then, the number of dimer-covering states in a $D$ subspace is

$$
\mathcal{N}_{D}(w)=\sum_{e_{0 I}^{D}}\left(e_{0 I}^{D}\left|\mathcal{K}_{1} \mathcal{K}_{2} \cdots \mathcal{K}_{L}\right| e_{0 I}^{D}\right) .
$$

Since $D_{n}=D_{n+1}$ for any dimer-covering singlet, $\mathcal{K}_{n}$ is a block-diagonal symmetric matrix that does not depend on $n$ and we can omit this subindex. For $L \rightarrow \infty$, the highest eigenvalue $\Lambda_{w D}$ of the $D$ block $\mathcal{K}_{D}$ dominates, and

$$
\mathcal{N}_{D}(w) \approx \Lambda_{w D}^{L} .
$$

Therefore,

$$
E_{D}(w) \approx w L \varepsilon_{0}+E_{r}(w, D) \approx w L \varepsilon_{0}-C L \ln \Lambda_{w D},
$$

where $\varepsilon_{0}=-0.375$ is the energy per spin of a single dimercovering configuration. Since ${ }^{31,32}$

$$
\Lambda_{w D}>\Lambda_{w D^{\prime}} \text { when }|D|<\left|D^{\prime}\right|,
$$

with $\Lambda_{w D}=\Lambda_{w|D|}$, the Heisenberg energy for the half-filled ground state belongs to the subspace $D=0$, as suggested by Eq. (5), and can be approximated (in units of $J$ ) by

$$
E_{0}(w) \approx w L \varepsilon_{0}-C L \ln \Lambda_{w 0} .
$$

$C$ is a fitting parameter independent of the structure to some degree. The value of $C$ for the nearest-neighbor isotropic Heisenberg model has been determined for a class of benzenoid hydrocarbons ${ }^{36}$ (with $C=0.5667$ ) and for finite square-lattice fragments ${ }^{38}$ (with $C=0.75$ ), by fitting the logarithm of the dimer-covering count to the resonance energy calculated from an equally weighted dimer-covering wave function. For the spin-1/2 square lattice, more general RVB approximations suggest ${ }^{31}$ a rough estimate of $C=0.94 \pm 0.19$. Here $C$ is fixed to yield a reasonably good estimate of the ground-state Heisenberg energy of the half-filled square lattice. Table I summarizes the ground-state energy per site for $w=4,6 \ldots, 20$ and its extrapolation to $w \rightarrow \infty$. We use $C=1$ from here on, since $C \approx 1.0083$ yields the ground-state energy of Liang et al. ${ }^{39}$

When adding a TSD to a $\mathrm{CuO}_{2}$ layer the transfer matrix $\mathcal{K}$ across the defect must be substituted by the appropriate $\mathcal{K}_{\mathbf{R}}$, where $\mathbf{R}$ is the vector position of the TSD. Therefore, the number of dimer-covering configurations when adding a pair of TSDs to the half-filled ground state, located, respectively, at $\mathbf{0}$ and $[m, j]$, with non-negative $m$ and $j$, with $m+j=$ odd, is
TABLE I. The ground-state resonance energy per site in units of $C J\left(-\ln \Lambda_{w 0} / w\right)$, and the extrapolation to $w \rightarrow \infty$. In the third column the ground-state energy per site in units of $J$ as obtained when taking $C=1$.

\begin{tabular}{ccc}
\hline \hline$w$ & $-\left(\ln \Lambda_{w 0}\right) / w$ & $\varepsilon_{0}$ \\
\hline 4 & -0.3292 & -0.7042 \\
6 & -0.3073 & -0.6823 \\
8 & -0.3001 & -0.6751 \\
10 & -0.2969 & -0.6719 \\
12 & -0.2953 & -0.6703 \\
14 & -0.2943 & -0.6693 \\
16 & -0.2936 & -0.6686 \\
18 & -0.2932 & -0.6682 \\
20 & -0.2929 & -0.6679 \\
$\infty$ & -0.2913 & -0.6664 \\
\hline \hline
\end{tabular}

$$
\begin{aligned}
\mathcal{N}_{[m, j]}(w) & =\left(\Lambda_{w 0}\left|\mathcal{K}_{0} \mathcal{K}^{m-1} \mathcal{K}_{[m, j]} \mathcal{K}^{L-m-1}\right| \Lambda_{w 0}\right) \\
& \approx \Lambda_{w 0}^{L-m-1}\left(\Lambda_{w 0}\left|\mathcal{K}_{0} \mathcal{K}^{m-1} \mathcal{K}_{[m, j]}\right| \Lambda_{w 0}\right) .
\end{aligned}
$$

Thence, the Heisenberg energy (in units of $J$ ) associated with a pair of static TSDs separated $[m, j], m+j=$ odd, with respect to the energy of the half-filled ground state is

$$
\varepsilon_{[m, j]}(w) \approx-2 \varepsilon_{0}+\ln \frac{\Lambda_{w 0}^{m+1}}{\left(\Lambda_{w 0}\left|\mathcal{K}_{0} \mathcal{K}^{m-1} \mathcal{K}_{[m, j]}\right| \Lambda_{w 0}\right)} .
$$

Table II summarizes the energies $\varepsilon_{[m, j]}(w)$ from $[m, j]$ $=[1,0]$ to $[7,4]$ and $w=4,6, \ldots, 20$. The $w \rightarrow \infty$ limit, $\varepsilon_{[m, j]}$, is obtained by fitting $\varepsilon_{[m, j]}(w)$ by a power series in $1 / w$.

For moderate to long distances, our results indicate that the Heisenberg energy of such a static excitation increases as $\sim \ln \rho$, as predicted in Ref. 28. Nevertheless, a tiny deviation from this behavior is observed for small values of $\rho$. This is because details of the lattice are more important for short distances, as also is expected from the form of the denominator in Eq. (14). See, for instance, Fig. 3. Therefore, it is expected that the TSDs of a pair will try to remain as close as possible. However, this is not enough to decide whether charge-wearing TSDs organize themselves as bound pairs of two charge-wearing TSDs, with bosonic character, or each charge-wearing TSD would bind to a spin-wearing TSD, leading to a fermionic-character pair.

\section{TWO-DIMENSIONAL EXTENDED WAVE FUNCTIONS}

The bosonic or the fermionic pairs are far from being static. The hopping terms of the Hamiltonian allow any charge-wearing TSD to move while the exchange part mixes up all the VB configurations. Therefore, the appropriate wave function must be a weighted superposition of all possible static configurations, fulfilling translational and point group symmetry conditions. Thence, the wave functions for both bosonic and fermionic extended pairs of TSDs should be invariant under the operations of the factor group isomorphic to the $C_{4 v}(4 \mathrm{~mm})$ group, as obtained by factorizing the 
TABLE II. Energy with respect to the half-filled ground state, $\varepsilon_{[m, j]}(w)$, of Eq. (14), associated with a pair of TSDs separated $[m, j]$, and the extrapolation to $w \rightarrow \infty, \varepsilon_{[m, j]}$. Values for $[0,1]$ and $[1,2]$ have been included to emphasize that $\varepsilon_{[j, m]}$ is asymptotically equivalent to $\varepsilon_{[m, j]}$

\begin{tabular}{|c|c|c|c|c|c|c|c|c|c|c|}
\hline$[m, j]$ & $w=4$ & 6 & 8 & 10 & 12 & 14 & 16 & 18 & 20 & $\infty$ \\
\hline$[1,0]$ & 2.3044 & 2.2064 & 2.1730 & 2.1587 & 2.1515 & 2.1473 & 2.1447 & 2.1429 & 2.1416 & 2.1334 \\
\hline$[0,1]$ & 1.9925 & 2.0708 & 2.1009 & 2.1143 & 2.1213 & 2.1254 & 2.1280 & 2.1298 & 2.1310 & 2.1386 \\
\hline$[2,1]$ & 2.8537 & 2.6837 & 2.6325 & 2.6133 & 2.6044 & 2.5996 & 2.5966 & 2.5947 & 2.5933 & 2.5792 \\
\hline$[1,2]$ & 2.5418 & 2.5481 & 2.5605 & 2.5689 & 2.5742 & 2.5776 & 2.5710 & 2.5816 & 2.5827 & 2.5845 \\
\hline$[3,0]$ & 3.2688 & 2.9316 & 2.8071 & 2.7508 & 2.7210 & 2.7034 & 2.6921 & 2.6845 & 2.6790 & 2.6509 \\
\hline$[3,2]$ & 3.3094 & 3.0050 & 2.9020 & 2.8594 & 2.8385 & 2.8268 & 2.8196 & 2.8148 & 2.8114 & 2.7900 \\
\hline$[4,1]$ & 3.7245 & 3.2529 & 3.0766 & 2.9969 & 2.9551 & 2.9307 & 2.9151 & 2.9045 & 2.8971 & 2.8617 \\
\hline$[4,3]$ & & 3.2657 & 3.1062 & 3.0416 & 3.0115 & 2.9956 & 2.9863 & 2.9804 & 2.9764 & 2.9481 \\
\hline$[5,0]$ & 4.1566 & 3.5239 & 3.2728 & 3.1525 & 3.0863 & 3.0461 & 3.0199 & 3.0018 & 2.9889 & 2.9304 \\
\hline$[5,2]$ & 4.1636 & 3.5393 & 3.2961 & 3.1823 & 3.1210 & 3.0845 & 3.0611 & 3.0452 & 3.0338 & 2.9866 \\
\hline$[6,1]$ & 4.5957 & 3.8103 & 3.4923 & 3.3379 & 3.2522 & 3.2000 & 3.1659 & 3.1424 & 3.1256 & 3.0553 \\
\hline$[5,4]$ & & & 3.3104 & 3.2115 & 3.1629 & 3.1365 & 3.1208 & 3.1108 & 3.1041 & 3.0701 \\
\hline$[6,3]$ & & 3.8129 & 3.5003 & 3.3521 & 3.2725 & 3.2255 & 3.1956 & 3.1756 & 3.1615 & 3.1078 \\
\hline$[7,0]$ & 5.0307 & 4.0860 & 3.6934 & 3.4970 & 3.3852 & 3.3155 & 3.2691 & 3.2369 & 3.2135 & 3.1048 \\
\hline$[7,2]$ & 5.0319 & 4.0892 & 3.6992 & 3.5056 & 3.3963 & 3.3287 & 3.2842 & 3.2534 & 3.2312 & 3.1366 \\
\hline$[6,5]$ & & & & 3.3598 & 3.2897 & 3.2518 & 3.2299 & 3.2163 & 3.2075 & 3.1633 \\
\hline$[7,4]$ & & & 3.7035 & 3.5159 & 3.4130 & 3.3513 & 3.3119 & 3.2853 & 3.2666 & 3.1980 \\
\hline
\end{tabular}

full group into the translational subgroup and the planar subgroup. Thence, there can be conceivable extended wave functions with symmetry $\mathcal{S}$,

$\mathcal{S}= \begin{cases}\mathcal{A}_{1}, & \text { totally symmetric }\left(x^{2}+y^{2}\right), \\ \mathcal{A}_{2}, & \text { antisymmetric under the four reflections, } \\ \mathcal{B}_{1}, & \text { antisymmetric under } C_{4}^{ \pm}, \sigma_{x \pm y}\left(x^{2}-y^{2}\right), \\ \mathcal{B}_{2}, & \text { antisymmetric under } C_{4}^{ \pm}, \sigma_{x}, \sigma_{y}(x y) .\end{cases}$

Therefore, symmetry-adapted extended wave functions for both the fermionic pairs and bosonic pairs can be written as

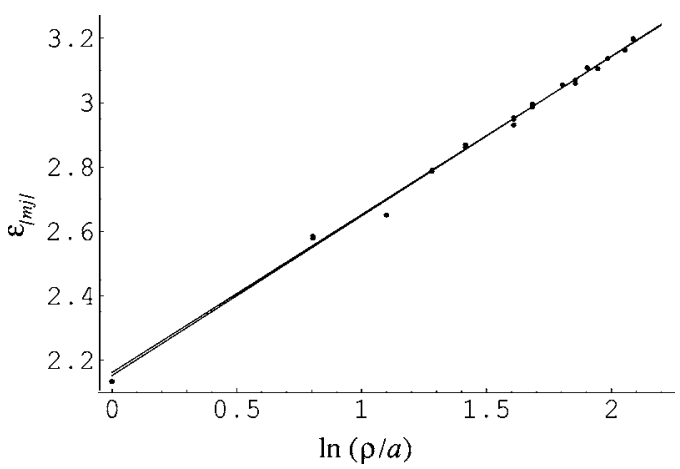

FIG. 3. Energy, $\varepsilon_{[m, j]}$, of Eq. (14) of a static pair of TSDs versus $\ln (\rho / a)$. The continuous lines are linear series approximations: (a) when all the points are retained $\left[\varepsilon_{[m, j]}=2.15235\right.$ $+0.495788 \ln (\rho / a)]$; (b) when $(\rho / a) \leqslant 3$ are not included $\left[\varepsilon_{[m, j]}\right.$ $=2.16149+0.490593 \ln (\rho / a)]$.

$$
\phi_{[m, j]}^{\mathcal{S}}(\mathbf{k}) \equiv N_{[m, j]} \sum_{\mathbf{R}}^{\in \mathcal{L}} e^{i \mathbf{k} \cdot \mathbf{R}} \sum_{\boldsymbol{\rho}_{[m, j]}}\left|\mathbf{R}, \mathbf{R}+\boldsymbol{\rho}_{[m, j]}\right\rangle \chi_{\boldsymbol{\rho}_{[m, j]}^{\mathcal{S}},}^{\mathcal{S}},
$$

where $N_{[m, j]}$ is the normalization; $\boldsymbol{\rho}_{[m, j]}$ is a vector obtained by any operation of the point group acting on $[m, j]$, with $0 \leqslant j<m$ and $m+j=$ odd; $\left|\mathbf{R}, \mathbf{R}+\boldsymbol{\rho}_{[m, j]}\right\rangle$ is the state with a static pair of TSDs, let's say, a charge-wearing TSD lying on site $\mathbf{R}$, and a spin-wearing TSD (a second charge-wearing TSD) on $\mathbf{R}+\boldsymbol{\rho}_{[m, j]}$ for the fermionic (bosonic) pairs; $\chi_{\boldsymbol{\rho}_{[m, j]}}^{\mathcal{S}}$ is the appropriate character of the irreducible representation $\mathcal{S}$, with $\chi_{[m, j]} \equiv 1$. Finally, $\mathcal{L}$ is the square lattice $(\mathcal{L}=\mathcal{A})$ for the fermionic (bosonic) pair. Then, care must be taken with the allowed values for k. For instance, when dealing with the fermionic pairs, $\mathbf{k}$ belongs to the Brillouin zone of a square lattice with lattice constant $a$. On the other hand, for bosonic pairs $\left|k_{x}\right|,\left|k_{y}\right| \leqslant \pi / 2 a$, because the summation is restricted to run on the sublattice $\mathcal{A}$.

It can be readily seen that only the $\mathcal{A}_{1}$ and $\mathcal{B}_{1}$ symmetries are allowed for $j=0$. Since different symmetries do not mix, here we restrict ourselves to $\mathcal{A}_{1}$ and $\mathcal{B}_{1}$ symmetries even when $j \neq 0$.

\section{A. Energy of the fermionic pairs}

The expectation values given by the wave functions of Eq. (16) are

$$
\begin{aligned}
\mathcal{S}_{[m, j]}^{\mathcal{S}}(\mathbf{k}) \approx & I-\frac{1}{2} t\left(\cos k_{x} a+\cos k_{y} a\right) \delta_{[m, j]}^{[1,0]}+\delta_{j, m-1}\left(1+\delta_{[m, j]}^{[1,0]}\right) \\
& \times t_{1}^{\prime} \chi_{[j, m]}^{\mathcal{S}} \cos k_{x} a \cos k_{y} a+J\left(\varepsilon_{[m, j]}+\gamma_{[m, j]}\right), \quad(17)
\end{aligned}
$$

where $I$ is the ionization potential (electron affinity) for hole- 


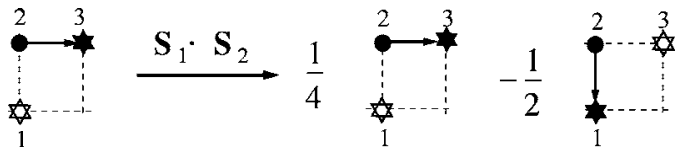

FIG. 4. The hopping of a spin-wearing TSD (white star) by the action of $H_{J}$.

doped (electron-doped) materials. $t$ and $t_{i}^{\prime}$ are nearest- and next-nearest-neighbor hopping integrals, with $i=1$ when there is only one charge-wearing TSD in the plaquette, and $i=2$ when there are two nearest-neighbor charge-wearing TSDs in the plaquette; $\gamma_{[m, j]}$ arises from the Heisenberg terms involving the spin-wearing TSD (see Fig. 4),

$$
\gamma_{[m, j]} \approx \begin{cases}\frac{3}{4}-\frac{1}{3} \chi_{[0,1]}^{\mathcal{S}}, & m=1, \\ 1-\frac{1}{8} \delta_{j, 1}-\frac{1}{4} \chi_{[j, m]}^{\mathcal{S}} \delta_{j, m-1}, & \text { otherwise. }\end{cases}
$$

There are two families of nonzero off-diagonal matrix elements of the Hamiltonian. When $m-m^{\prime}= \pm 1$ and $j-j^{\prime}$ $= \pm 1$,

$$
H_{[m, j]}^{\left[m^{\prime}, j^{\prime}\right]}=\lambda\left(t_{1}^{\prime} \cos k_{x} a \cos k_{y} a-\frac{1}{4} J-\frac{1}{8} J \delta_{j, m-1} \chi_{[j, m]}^{\mathcal{S}}\right) .
$$

When $m^{\prime}-m= \pm 2$ and $j^{\prime}=j$ or $m^{\prime}=m$ and $j^{\prime}-j= \pm 2$,

$$
H_{[m, j]}^{\left[m^{\prime}, j^{\prime}\right]}= \begin{cases}-\sqrt{3} J / 12, & {\left[m^{\prime}, j^{\prime}\right] \text { or }[m, j]=[1,0],} \\ -\lambda J / 8, & \text { otherwise, }\end{cases}
$$

with $\lambda=\sqrt{2}$ when either $j$ or $j^{\prime}$ is zero (but not both), and $\lambda=1$ otherwise.

The zero-order lowest-lying fermionic pairs are the $[1,0]$. Close to $\Gamma$ the energy of these fermionic pairs is

$$
\mathcal{S}_{[1,0]}^{\mathcal{S}}(\mathbf{k}) \approx \zeta_{\Gamma}^{\mathcal{S}}+\frac{\hbar^{2} k_{\|}^{2}}{2 m_{\|}^{\mathcal{S}}}
$$

with

$$
\begin{aligned}
\mathcal{\zeta}_{\Gamma}^{\mathcal{S}} & \equiv I-t+2 t_{1}^{\prime} \chi_{[0,1]}^{\mathcal{S}}+J\left(\varepsilon_{[1,0]}+\frac{3}{4}-\frac{1}{3} \chi_{[0,1]}^{\mathcal{S}}\right), \\
\frac{\hbar^{2}}{2 m_{\|}^{\mathcal{S}}} & \equiv\left(\frac{1}{4} t-t_{1}^{\prime} \chi_{[0,1]}^{\mathcal{S}}\right) a^{2} .
\end{aligned}
$$

Thence, for $t_{1}^{\prime}>J / 6$, the zero-order lowest-lying band has $x^{2}-y^{2}$ symmetry. This is the case for the $\mathrm{La}_{2-x} \mathrm{Sr}_{x} \mathrm{CuO}_{4}$ (Ref. 23) (LSCO). For the monolayered HTSC of Table 1 in Ref. 22 we know that $J / 6 \sim 0.019-0.030 \mathrm{eV}$. On the other hand, strong differences on hopping integrals among the different HTSC are not expected, as suggested by the small variations observed on the nearest-neighbor hopping integral, $t$. Therefore, we expect that the zero-order lowest-lying band will show $\mathcal{B}_{1}$ symmetry for all of these HTSC.

We are now concerned whether admixing wave functions with different $[m, j]$ to the $\phi_{[1,0]}^{\mathcal{S}}(\mathbf{k})$ would be relevant or even if the ordering of the lowest-lying $\mathcal{A}_{1}$ and $\mathcal{B}_{1}$ bands could be reversed. We have obtained the corrections to $\zeta_{[1,0]}^{\mathcal{A}_{1}}(\mathbf{k})$ and $\zeta_{[1,0]}^{\mathcal{B}_{1}}(\mathbf{k})$ by diagonalizing the matrix of the Hamiltonian in the basis of the two, three, and four lowest- lying wave functions with the appropriate $\mathcal{A}$ or $\mathcal{B}$ symmetries. Thence, making use of the parameters for the LSCO, ${ }^{23}$ when the number of fermionic pairs per $\mathrm{Cu}$ is $p$ $=0.05-0.07$ we obtain corrections to the zero-order Fermi energy of $\Delta^{(2)}=-16.98$ to $-14.56 \mathrm{meV}, \quad \Delta^{(3)}=-1.45$ to $-1.33 \mathrm{meV}$, and $\Delta^{(4)}=-0.26$ to $-0.20 \mathrm{meV}$ for the $\mathcal{B}_{1}$ band, while for the $\mathcal{A}_{1}$ the corrections are $\Delta^{(2)}=-7.21$ to $-5.1 \mathrm{meV}, \Delta^{(3)}=-3.15$ to $-2.7 \mathrm{meV}$, and $\Delta^{(4)}=-0.15$ to $-0.1 \mathrm{meV}$. For the monolayered HTSC of Table 1 in Ref. 22, assuming $t_{1}^{\prime} \sim 0.2 t$, these corrections are slightly decreasing with $J / t$. We observe that, up to $\mathrm{meV}$, the correction to the $\mathcal{A}_{1}$ energy is smaller than the correction to the $\mathcal{B}_{1}$. Therefore, it is expected that the lowest-lying band still has $x^{2}-y^{2}$ symmetry. Furthermore, the band with symmetry $\mathcal{A}_{1}$ would not start filling until a critical doping of $p \approx 0.20-0.22$ holes per $\mathrm{CuO}_{2}$ unit, provided that all the charge-wearing TSDs organize as fermionic pairs. At this doping, the corrections $\Delta^{(n)}$ to the energies are still smaller than those referred above. Since this doping is out of the range of our interest, we restrict ourselves to consider only the band with $x^{2}-y^{2}$ symmetry. Also, since the error in the parameters of the Hamiltonian are of the order of $\mathrm{meV}$, we neglect corrections to the energy smaller than $1 \mathrm{meV}$. Therefore, we consider only the [1, 0], $[2,1]$, and $[3,0]$ wave functions to describe the lowest-lying band of the fermionic pairs.

\section{B. Energy of the bosonic pairs}

When dealing with $t$-J-like model Hamiltonians, the electrostatic repulsion is generally neglected, although with some exceptions. ${ }^{40,41}$ Since the screened electrostatic repulsion between the charge-wearing TSDs in a pair, $V_{[m, j]}$, may be relevant, here it is included in the diagonal terms of the Hamiltonian,

$$
\langle H\rangle_{[m, j]}^{\mathcal{S}}=J \varepsilon_{[m, j]}+2 I+V_{[m, j]}+\left\langle H_{t}+H_{t^{\prime}}\right\rangle_{[m, j]}^{\mathcal{S}} .
$$

It is not difficult to obtain $\left\langle H_{t}\right\rangle_{[m, j]}^{\mathcal{S}}=0$, and

$$
\left\langle H_{t^{\prime}}\right\rangle_{[m, j]}^{\mathcal{S}}=\kappa_{[m, j]} \chi_{[j, m]}^{\mathcal{S}}\left(1+\cos k_{x} a \cos k_{y} a\right),
$$

with

$$
\kappa_{[m, j]}=\left\{\begin{array}{ll}
2 t_{2}^{\prime}, & {[m, j]=[1,0],} \\
t_{1}^{\prime}, & m-j=1, \\
0, & \text { otherwise. }
\end{array} \quad m>1,\right.
$$

The electrostatic repulsion within a bosonic pair is expected to be screened by the gas of the fermionic pairs. However, the fermionic pairs have been considered so far as moving in a two-dimensional square lattice. It is generally accepted that the $c$ axis effect is simply to tune the electronic structure of the $\mathrm{CuO}_{2}$ planes. Nevertheless, screening is a three-dimensional effect that could be taken into account by an interlayer hopping integral $t_{\perp}$. Considering a nonzero $t_{\perp}$ would imply a correction to the energy $\approx t_{\perp} k_{\perp}^{2} c^{2}$, where $c$ is the lattice constant perpendicular to the $a b$ layers. Since $t_{\perp}$ is rather small, ${ }^{42}$ it can be neglected for the energy-balance considerations, but it is essential for screening purposes. Then, for the electrostatic repulsion within a bosonic pair we 
take as a first approximation the Yukawa potential ${ }^{34,35}$ as the dominant term,

$$
V_{\rho} \approx \frac{q^{2}}{\rho} \exp \left[-\left(4 \pi e^{2} g_{F}\right)^{1 / 2} \rho\right],
$$

where $g_{F}$ is the density of states at the Fermi level of the fermionic pairs per unit of volume of the solid,

$$
g_{F} \approx\left(\frac{3 m_{\perp} m_{\|}^{2} \nu p}{\pi^{4} \hbar^{6} a^{2} c}\right)^{1 / 3}
$$

$p$ being the number of fermionic pairs per site, and $\nu$ is the number of square-lattice layers cutting a unit cell. Therefore, close to $\Gamma$, the diagonal terms of the Hamiltonian are

$$
\begin{aligned}
\langle H\rangle_{[m, j]}^{\mathcal{S}} \approx & J \varepsilon_{[m, j]}+2 I+\frac{e^{2}}{\rho} \exp \left[-\beta \rho(\nu p)^{1 / 6}\right] \\
& +\kappa_{[m, j]} \chi_{[j, m]}^{\mathcal{S}}\left(2-\frac{1}{2} a^{2} k^{2}\right)
\end{aligned}
$$

with

$$
\beta \approx \frac{2 e}{a}\left(\frac{6}{\pi t_{\perp}\left(t+4 t_{1}^{\prime}\right)^{2} c^{3}}\right)^{1 / 6} .
$$

The nonzero off-diagonal elements of the Hamiltonian can also be readily obtained,

$$
H_{[m, j]}^{\left[m^{\prime}, j^{\prime}\right]}=\lambda t_{1}^{\prime}\left(1+\cos k_{x} a \cos k_{y} a\right), \quad\left|m-m^{\prime}\right|,\left|j-j^{\prime}\right|=1,
$$

with $\lambda=\sqrt{2}$ when either $j$ or $j^{\prime}$ is zero, and $\lambda=1$ otherwise.

Since the nonzero off-diagonal elements of the Hamiltonian are important as compared to the differences among the diagonal elements, the energy of the bosonic pairs must be obtained by diagonalizing the matrix of the Hamiltonian.

Since the screened electrostatic repulsion decays faster than exponentially, while $\varepsilon_{[m, j]}$ increases logarithmically, there must be a minimum in the energy and confinement is expected to occur. Furthermore, from Eq. (28) and assuming that $t_{1}^{\prime}, t_{2}^{\prime}>0,{ }^{23}$ it is expected that the bosonic pairs will also show $x^{2}-y^{2}$ symmetry as it is generally accepted. ${ }^{3}$ At this point, it is worth noting that if the next-nearest-neighbor hopping is neglected and the Hamiltonian is reduced to the $t$ - $J$ model, it turns out that the $\mathcal{A}_{1}$ and $\mathcal{B}_{1}$ symmetries would be degenerate.

\section{Two-fluids equilibrium condition}

At $T=0$, the question now is whether this lowest-lying bosonic pairs would have lower energy than two fermionic pairs in its Fermi level, so the bosonic pairs would be favored. At low doping level, it is expected that the fermionic pairs will be favored. Nevertheless, the Fermi level, $k_{F}$ $\approx \sqrt{2 \pi p} / a$, increases linearly with $p$, while the electrostatic repulsion among the two charge-wearing TSDs in the bosonic pair is exponentially reduced with $p^{1 / 6}$, so its ground state energy is lowered. Therefore, we wonder whether there

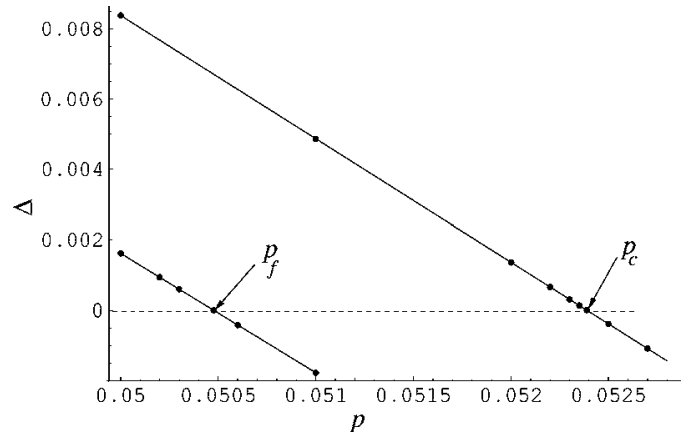

FIG. 5. The ground-state energy of the bosonic pairs, as measured respect twice the energy of a fermionic pair at its Fermi level, $\Delta(p)$. Top, $\Delta(p)$, with screened electrostatic repulsion. Bottom, $\Delta_{0}(p)$, without electrostatic repulsion.

exist a critical value $p_{c}$ such that the ground state of the bosonic pairs, as measured with respect twice the energy of a fermionic pair at its Fermi level, $\Delta(p)$, is zero.

To explore such a possibility, we have diagonalized the matrix of the Hamiltonian for $\mathbf{k}=0$, and increasing values of $p$. To reach corrections to the ground-state energy within the order of $\mathrm{meV}$, we have considered up to a $12 \times 12$ matrix involving all the states which would contribute to perturbation theory truncated to tenth order. For a certain regime of parameters, at low enough doping level, the fermionic pairs are favorable. As $p$ increases there exist a critical value of doping, $p_{c}$, such that $\Delta\left(p_{c}\right)=0$ for the lowest-lying bosonic pairs.

Doping above $p_{c}$ yields bosonic pairs. In this case, the bosonic pairs are expected also to contribute to the screening and the electrostatic repulsion could become negligible. If so, there would be a cascade process of pairing among the fermionic pairs until a new equilibrium between the two fluids is reached at $p_{f}<p_{c}$. Thence, we expect the number of bosonic pairs at $p>p_{f}$ to be $p_{b} \approx\left(p-p_{f}\right) / 2$. There is a lower limit of $p_{f}$ such that $\Delta_{0}\left(p_{f}\right)=0$, as obtained when the electrostatic repulsion is completely neglected.

For the sake of estimating the order of magnitude of $p_{c}$ and $p_{f}$ for a generic HTSC, let us make use of the parameters appropriate for LSCO. We take $a \approx 3.8 \AA$ and $c / a \approx 3.47$ from Ref. 3. For the Hamiltonian parameters, we take $J$ $=0.144 \mathrm{eV}$ and $t=0.549 \mathrm{eV}$ from Ref. 22, and $t_{2}^{\prime}=0.130 \mathrm{eV}$ and $t_{1}^{\prime}=0.112 \mathrm{eV}$ from Ref. 23. All of these parameters were obtained from high-level $a b$ initio calculations with the geometry as the only external input, being the errors within the $\mathrm{meV}$. Since there is no high-level ab initio calculation for the interlayer hopping integral, we use the low-doping $t_{\perp}$ $\approx 0.7 \mathrm{meV}$ obtained from experimental results by Zha, Cooper, and Pines. ${ }^{42}$ Within this parameter regime we get $\beta \approx 7.8 / a$. Computing $\Delta(p)$ for increasing values of $p$ we find that $\Delta(p)$ is changing its sign at $p_{c} \approx 0.0524$ (see, for instance, Fig. 5). At $p_{c}$, the mean distance between the two holes of the pair is $\left\langle\rho_{c}\right\rangle \approx 9.08 \AA$ with a standard deviation $\sigma_{c} \approx 5.08 \AA$. Identifying in a rather loose way the spatial extent of the pair wave function $\left(\sim \rho_{c}+\sigma_{c}\right)$ with the coherence length $\xi$, we obtain $\xi \approx 14.16 \AA$, in good agreement with the in-plane value $(\xi \sim 14-15 \AA$ ) (Refs. 2 and 3 ) suggested from experimental findings. 


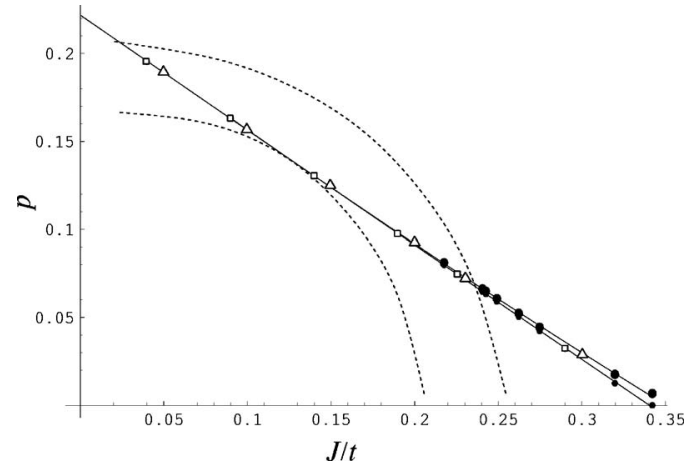

FIG. 6. $p_{c}$ for the monolayered HTSC $(\bullet)$ and for LSCO with different $J / t(\triangle) ; p_{f}$ for the monolayered HTSC $(\bullet)$ and for LSCO with different $J / t(\square)$. The continuous lines are power series approximations to $p_{c}$ and $p_{f}$ sets. The dotted lines are hypothetical $J / t$ vs $p$ curves.

On the other hand, when the electrostatic repulsion is neglected, $\Delta_{0}(p)$ changes its sign at $p_{f} \approx 0.0505$, and the mean distance between the two holes is $\left\langle\rho_{f}\right\rangle \approx 8.78 \AA$ with a standard deviation $\sigma_{f} \approx 4.99 \AA$. Therefore, the estimated coherence length is $\xi \approx 13.77 \AA$. Again, it is worth noting that if the next-nearest-neighbor hopping is neglected and the Hamiltonian is reduced to the $t$ - $J$ model, the value of the critical doping $\left(p_{c} \approx 0.28\right)$ is out of the range where the superconductivity is observed. In addition, at so high doping the validity of such model Hamiltonians could be questioned.

As far as we know, for other monolayered cuprate superconductors only the $t$ and $J$ parameters have been obtained from high-level $a b$ initio calculations. Nevertheless, for the purpose of estimating how $p_{c}$ and $p_{f}$ vary with $J / t$, let us assume that the ratios $t_{1}^{\prime} / t$ and $t_{2}^{\prime} / t$, as well $a \beta$ do not change very much among them, and use the values appropriate for LSCO. If so, we find that $p_{f}$ and $p_{c}$ increase as $J / t$ decreases. See, for instance, Fig. 6, where we also include the $p_{f}$ and $p_{c}$ values for LSCO as a function of $J / t$. This result suggests that for low $J / t$ the onset of superconductivity would be located at a too high level of doping such that it could be beyond the validity of the present approximation. Therefore, the superconductivity could be suppressed.

Furthermore, it is worth noting here that the parameters that characterize a superconductor are taken as independent of doping. Nevertheless, $J$ as well as the hopping integral do depend locally on doping, as suggested by the high-level $a b$ initio calculations of Calzado and Malrieu. ${ }^{23}$ For instance, their calculations suggest that $J$ decreases, while $t$ increases, with doping. Therefore, it is expected that $J / t$ decreases with doping, probably not linearly (see, for instance, the dotted lines of Fig. 6). Consequently, $p_{b}$ would decrease, eventually down to zero at a critical doping $p_{c}^{\prime}$ such that the $J / t$ as a function of the doping crosses again the $p_{f}$ function. Thence, the superconductivity would be suppressed in the overdoped regime for a doping $p>p_{c}^{\prime}$. Therefore, a better knowledge of the parameters is essential to fully understand the phenomenon of HTSC.

\section{SUMMARY AND CONCLUSIONS}

We have shown that the Heisenberg energy associated with a pair of TSD in a spin $=1 / 2$ square lattice increases logarithmically with distance. Therefore, a charge-wearing TSD (either a hole or a doubly occupied site) in a spin $=1 / 2$ square lattice binds to another TSD, either to a spinwearing TSD or to another charge-wearing TSD.

We have constructed symmetry-adapted extended wave functions for both a fermionic pair of a charge-wearing TSD and a spin-wearing TSD, and a bosonic pair of two chargewearing TSD. The energy associated with such fermionic and bosonic pairs has been obtained.

For the lowest-lying fermionic band and the lowest-lying bosonic pairs the symmetry turns to be $x^{2}-y^{2}$ when $t_{1}^{\prime}$ $>J / 6$ and $t_{1}^{\prime}, t_{2}^{\prime}>0$, respectively. Since these conditions are fulfilled for monolayered HTSC, we obtain that the symmetry of the bosonic pairs is $x^{2}-y^{2}$ for these materials, as it is generally accepted. ${ }^{3}$

For the LSCO compound, we find a critical doping for bosonic pairing $p_{c} \approx 0.0524$ and $p_{f} \approx 0.0505$ (when the electrostatic repulsion is completely neglected). This finding could be related to the onset of high- $T_{c}$ superconductivity, the superconducting state being a Bose condensate. This is also compatible with the existence of pairs above $T_{c}$, a forerunner of the pseudogap physics of the cuprates. At the critical doping, we find a mean distance between the two holes of the pair $\left\langle\rho_{c}\right\rangle \approx 9.08 \AA\left(\left\langle\rho_{f}\right\rangle \approx 8.78 \AA\right)$, and an estimated coherence length $\xi \approx 14.16 \AA(\xi \approx 13.77 \AA)$. These features are in good agreement with the experimental result of $p_{c} \approx 0.05$ (Ref. 43) and $\xi \sim 14-15 \AA .{ }^{2,3}$

For the monolayered cuprate superconductors of Table 1 in Ref. 22, we have obtained $p_{f}$ and $p_{c}$ as a function of $a$ and $J / t$, while keeping fixed $a \beta \approx 7.8$ and $t_{i}^{\prime} / t$. See, for instance, Fig. 6. It can be observed that $p_{f}$ and $p_{c}$ increase as $J / t$ is lowered.

Extensions of the present work towards $T \neq 0$, and to other possible charge-wearing TSDs self-organization are in progress. For instance, it is of interest to explore the parameter regime for bosonic pairs with $x y$ symmetry, phase separation, and stripe formation.

\section{ACKNOWLEDGMENTS}

This research was supported by the Spanish DGI (Project No. PFM2002-02629). The author thanks Professor D.J. Klein, Professor A. Labarta, Professor F. Illas, and Dr. I. de P. R. Moreira for valuable suggestions. 
*Electronic address: m.angels.garcia_bach@ub.edu

${ }^{1}$ J. G. Bednorz and K. A. Müller, Z. Phys. B: Condens. Matter 64, 188 (1986).

${ }^{2}$ T. Barnes, Int. J. Mod. Phys. C 2, 659 (1991).

${ }^{3}$ E. Dagotto, Rev. Mod. Phys. 66, 763 (1994).

${ }^{4}$ J. Orenstein and A. J. Mills, Science 288, 468 (2000).

${ }^{5}$ Nai-Chang Yeh, cond-mat/0210656.

${ }^{6}$ M. R. Norman and C. Pépin, Rep. Prog. Phys. 66, 1547 (2003).

${ }^{7}$ J. P. Perdew, in Electronic Structure of Solids, edited by P. Ziesche and H. Eschrig (Akademic Verlag, Berlin, 1991).

${ }^{8}$ W. E. Pickett, Rev. Mod. Phys. 61, 433 (1989); 61, 749(E) (1989).

${ }^{9}$ I. de P. R. Moreira and R. Dovesi, Int. J. Quantum Chem. 99, 805 (2004).

${ }^{10}$ R. W. Godby, M. Schluter, and L. J. Sham, Phys. Rev. Lett. 56, 2415 (1986).

${ }^{11}$ M. S. Hybertsen and S. G. Louie, Phys. Rev. B 34, 5390 (1986).

${ }^{12}$ A. Svane and O. Gunnarsson, Phys. Rev. Lett. 65, 1148 (1990).

${ }^{13}$ A. Svane, Phys. Rev. Lett. 68, 1900 (1992).

${ }^{14}$ Z. Szotek, W. M. Temmerman, and H. Winter, Phys. Rev. B 47, 4029 (1993).

${ }^{15}$ M. T. Czyzyk and G. A. Sawatzky, Phys. Rev. B 49, 14211 (1994).

${ }^{16}$ V. I. Anisimov, M. A. Korotin, J. Zaanen, and O. K. Andersen, Phys. Rev. Lett. 68, 345 (1992).

${ }^{17}$ Pan Wei and Zheng Qing Qi, Phys. Rev. B 49, 12159 (1994).

${ }^{18}$ P. W. Anderson, Science 235, 1196 (1987).

${ }^{19}$ V. J. Emery, Phys. Rev. Lett. 58, 2794 (1987).

${ }^{20}$ V. J. Emery and G. Reiter, Phys. Rev. B 38, 4547 (1988).

${ }^{21}$ F. C. Zhang and T. M. Rice, Phys. Rev. B 37, 3759 (1988).

${ }^{22}$ I. de P. R. Moreira, D. Muñoz, F. Illas, C. de Graaf, and M. A. Garcia-Bach, Chem. Phys. Lett. 345, 183 (2001).

${ }^{23}$ C. J. Calzado and J.-P. Malrieu, Phys. Rev. B 63, 214520 (2001).

${ }^{24}$ D. Muñoz, I. de P. R. Moreira, and F. Illas, Phys. Rev. B 65, 224521 (2002).
${ }^{25}$ E. H. Lieb and D. C. Mattis, J. Math. Phys. 3, 749 (1962).

${ }^{26}$ S. A. Kivelson, D. S. Rokhsar, and J. P. Sethna, Phys. Rev. B 35, 8865 (1987).

${ }^{27}$ S. Kivelson, Phys. Rev. B 36, 7237 (1987).

${ }^{28}$ D. J. Klein, T. G. Schmalz, M. A. Garcia-Bach, R. Valentí, and T. P. Živković, Phys. Rev. B 43, 719 (1991).

${ }^{29}$ D. J. Klein, T. P. Živković, and R. Valentí, Phys. Rev. B 43, 723 (1991).

${ }^{30}$ T. H. Hansson, V. Oganesyan, and S. L. Sondhi, Ann. Phys. (N.Y.) 313, 497 (2004).

${ }^{31}$ M. A. Garcia-Bach, Eur. Phys. J. B 14, 439 (2000).

${ }^{32}$ M. A. Garcia-Bach, "Many-body VB ansätze. From polymers and ladder materials to the square lattice," Valence Bond Theory (Elsevier, New York, 2002), pp. 729-768.

${ }^{33}$ M. S. Hybertsen, E. B. Stechel, M. Schluter, and D. R. Jennison, Phys. Rev. B 41, 11068 (1990).

${ }^{34}$ N. W. Ashcroft and N. D. Mermin, Solid State Physics (HoltSaunders, Japan, 1981), pp. 337-342.

${ }^{35}$ O. Madelung, Introduction to Solid-State Theory, Springer Series in Solid State Sciences, Vol. 2 (Springer-Verlag, Berlin, 1978), pp. 114-117.

${ }^{36}$ W. A. Seitz, D. J. Klein, T. G. Schmalz, and M. A. Garcia-Bach, Chem. Phys. Lett. 115, 139 (1985); 118, 110(E) (1985).

${ }^{37}$ D. J. Klein, G. E. Hite, and T. G. Schmalz, J. Comput. Chem. 7, 443 (1986).

${ }^{38}$ T. P. Živković, B. L. Sandleback, T. G. Schmalz, and D. J. Klein, Phys. Rev. B 41, 2249 (1990).

${ }^{39}$ S. Liang, B. Doucot, and P. W. Anderson, Phys. Rev. Lett. 61, 365 (1988).

${ }^{40}$ S. A. Kivelson, V. J. Emery, and H. Q. Lin, Phys. Rev. B 42, 6523 (1990).

${ }^{41}$ E. Dagotto and J. Riera, Phys. Rev. B 46, 12084 (1992).

${ }^{42}$ Y. Zha, S. L. Cooper, and D. Pines, Phys. Rev. B 53, 8253 (1996).

${ }^{43}$ K. Yamada et al., Phys. Rev. B 57, 6165 (1998). 